\title{
Effects of treatment with sitagliptin on hepatotoxicity induced by acetaminophen in mice
}

\author{
Wafaa Ahmed Hewedy ${ }^{\left(11^{*}\right.}$ \\ ${ }^{I}$ Department of Clinical Pharmacology, Faculty of Medicine, Alexandria University, Alexandria, Egypt
}

\begin{abstract}
Up to date, the management of hepatotoxicity induced by a suicidal or unintentional overdose of acetaminophen (APAP) remains a therapeutic challenge. The present study aimed to elucidate the potential effect of sitagliptin, a DPP-4 inhibitor, to ameliorate the acute injurious effects of acetaminophen on the liver. APAP toxicity was induced in mice by an intraperitoneal injection of APAP $(400 \mathrm{mg} / \mathrm{kg})$. The effect of treatment with sitagliptin, initiated 5 days prior to APAP injection, was evaluated. Serum indices of hepatotoxicity, oxidative stress markers in liver tissues, serum IL-1 $\beta$, and TNF- $\alpha$ in addition to hepaticNF-E2-related factor-2 (Nrf2) were determined. Our results showed that APAP induced marked hepatic injury as evidenced by an increase in serum levels of ALT and AST, in addition to the deterioration of histological grading. Oxidative stress markers, serum TNF- $\alpha$, and IL-1 $\beta$ were also elevated. Sitagliptin successfully ameliorated the histological changes induced by APAP, improving liver function tests and liver oxidant status accompanied with a marked increase in Nrf2 level in hepatic tissues. Thus, the hepatoprotective effects of sitagliptin in this animal model seem to involve Nrf2 modulation, coincidental with its anti-inflammatory and antioxidant effects.
\end{abstract}

Keywords: Acetaminophen. Dipeptidyl peptidase-4 inhibitors. Hepatotoxicity. Nrf2. Sitagliptin.

\section{INTRODUCTION}

Acetaminophen ( $N$-acetyl- $p$-aminophenol; APAP) overdose is considered as a serious and silent killer. It may initially present with few, if any, symptoms that may progress into acute liver failure and death if not treated promptly (Lancaster, Hiatt, Zarrinpar, 2015). Chronic liver injury is also recognized in some patients on long-term APAP treatment (Watelet et al., 2007). The safe limit of APAP for therapeutic indications has been reported to be variable among individuals. Moreover, malnutrition, chronic alcohol misuse, and nonalcoholic fatty liver disease (NAFLD) are recognized as risk factors for APAP-induced hepatotoxicity (Michaut et al., 2014).

$N$-Acetylcysteine remains the gold standard for acute APAP-hepatotoxicity (Lancaster, Hiatt, Zarrinpar,

*Correspondence: W. A. Hewedy, Department of Clinical Pharmacology, Faculty of Medicine, Alexandria University Alexandria, Egypt. E-mail: drwafaa05@yahoo.com; wafaa.hewedy@alexmed.edu.eg
2015). However, once acute liver failure is developed, the role of NAC is questionable. Therefore, efforts in developing novel treatments for APAP-induced hepatotoxicity, with more potency than $N$-acetyl cysteine, are warranted.

Therapeutic doses of APAP are usually metabolized in the liver by glucuronidation and sulfation. Less than $5 \%$ is oxidized by the microsomal cytochrome $\mathrm{P} 450$ system to $N$-acetyl- $p$-benzoquinone imine (NAPQI), a highly reactive metabolite. NAPQI is then conjugated and detoxified with glutathione (GSH) in a reaction catalyzed by glutathione $S$-transferases (GSTs) to produce harmless metabolites (McGill, Jaeschke, 2013). Toxic doses of APAP, however, will lead to an excessive generation of NAPQI and depletion of GSH. Excess NAPQI interacts with specific mitochondrial proteins forming NAPQI adducts with increased superoxide and peroxynitrite production that correlates with hepatic toxicity (Jaeschke, Knight, Bajt, 2003; McGill, Jaeschke, 2013). An inflammatory reaction characterized by activation of transcription factors, such as nuclear factor 
kappa B (NF- $\kappa \mathrm{B})$, regulates the expression of several proinflammatory genes (e.g., tumor necrosis factor- $\alpha$ (TNF $\alpha$ ), interleukin-1 $\beta$ (IL-1 $\beta$ ), and cyclooxygenase-2), each of which has been implicated in hepatotoxicity (Dambach et al., 2006).

In the last decade, nuclear erythroid 2-related factor 2 (Nrf2) emerges as an important regulator of cellular resistance to oxidants (Lee et al., 2005). Under normal conditions, Nrf2 is suppressed by Kelch-like ECH associated protein (Keap1). Activation of Nrf2 signaling pathway occurs in a state of oxidative stress via modification of critical cysteine thiols of Keap1 and Nrf2 and release of Nrf2 from its cytoplasmic Keap1. In the nucleus, Nrf2 induces the expression of several cytoprotective genes possessing an antioxidant response element (ARE) in the promoter region. Nrf2 pathway activation could alleviate oxidative insult in multiple organs. The liver is particularly susceptible to a wide array of insults; thus, the role of Nrf2 activation in many liver diseases had been thoroughly investigated (Shin, Yang, Ki, 2013).

Dipeptidyl peptidase-4 (DPP-4) inhibitors are currently used for the treatment of type 2 diabetes mellitus (T2DM) (Mulvihill, Drucker, 2014). Recently, DPP-4 inhibitors also provide an alternative therapeutic option for the treatment of NAFLD in T2DM (PortilloSanchez, Cusi, 2016). Sitagliptin, a member of DPP-4 inhibitors, was found to exert a hepatoprotective effect in some animal models of liver injuries (Abo-Haded et al., 2017; Jung et al., 2014). However, the exact hepatoprotective effects of sitagliptin are complex and not yet completely clarified. It was found to modulate lipid metabolism and exert anti-inflammatory and antioxidant effects.

In view of the increasing use of DPP4 inhibitors, the aim of this study was to investigate the possible hepatoprotective effect of sitagliptin, a DPP-4 inhibitor, in an animal model of APAP-induced hepatotoxicity and to elucidate the underlying mechanisms.

\section{MATERIAL AND METHODS}

\section{Animals}

Male BALB/C mice weighing 18-22 $\mathrm{g}$ were used in this study. Mice were housed in groups of five and kept in cages with standard conditions of temperature and humidity. They were fed standard food ad libitum with free access to water; however, they were fasted $16 \mathrm{~h}$ prior to treatment with APAP in order to deplete GSH stores.
The experiments comply with ARRIVE guidelines of Animal Care and were carried out in accordance with the National Institutes of Health Guide for the Care and Use of Laboratory Animals. The study protocol was approved by the Local Ethical Committee of the Faculty of Medicine, Alexandria University.

\section{Drugs and chemicals}

Acetaminophen (APAP), obtained from the Egyptian International Pharmaceutical Industries Company (EIPICO, 10 $10^{\text {th }}$ of Ramadan, Egypt), and sitagliptin (Januvia tablets ${ }^{\circledR}$, Merck Sharp, and Dohme) were used. All other chemicals used in the study were of analytical grade and were obtained from local commercial sources. APAP was dissolved in warm saline solution $\left(40{ }^{\circ} \mathrm{C}\right)$ to give a final concentration of $16 \mathrm{mg} / \mathrm{mL}$. The prepared APAP solution was administered to the mice as $25 \mathrm{~mL} / \mathrm{kg}$. Sitagliptin was freshly dissolved in normal saline at a final concentration of $0.4 \mathrm{mg} / \mathrm{mL}$ and administered through oral gavage in a constant volume of $25 \mathrm{~mL} / \mathrm{kg}$.

\section{Experimental design}

Mice were randomly divided into four groups, 10 mice each: group I, normal control group; group II, APAP-treated group; group III, sitagliptin-treated group; group IV, $N$-acetylcysteine-treated group.

Groups I, II, and IV received $1 \mathrm{~mL}$ of normal saline/day orally for 5 days, while group III received sitagliptin $(10 \mathrm{mg} / \mathrm{kg} /$ day $)$ for 5 days. On day 6 , APAP hepatotoxicity was induced in groups II-IV by a single intraperitoneal (i.p.) injection of $400 \mathrm{mg} / \mathrm{kg}$ APAP (Shimizu et al., 2014). Group I received an equal amount of saline. Three hours later, mice in group III received an additional dose of $10 \mathrm{mg} / \mathrm{kg}$ sitagliptin and group IV was treated with $N$-acetylcysteine $100 \mathrm{mg} / \mathrm{kg}$ orally, while group II received vehicle only.

Twenty-four hours after APAP injection, all mice were anesthetized with an intraperitoneal injection of ketamine-xylazine (50 and $8 \mathrm{mg} / \mathrm{kg}$, resp.). Blood samples were collected from the abdominal aorta, centrifuged at $2500 \mathrm{~g}$ for $10 \mathrm{~min}$ for separation of serum, aliquoted, and stored at $-20{ }^{\circ} \mathrm{C}$. Liver tissues were dissected, washed with ice-cold saline, and divided into two parts. One part was immediately stored at $-80{ }^{\circ} \mathrm{C}$ for further assays. The other part was fixed in formalin for histological examination. 


\section{Biochemical studies}

\section{Determination of serum indices of hepatotoxicity}

Serum activities of alanine aminotransferase (ALT) and aspartate aminotransferase (AST) were assayed according to the previously described method (Reitman, Frankel, 1957), using commercially available colorimetric kits (Spectrum Diagnostics, Cairo, Egypt). Results were read at $505 \mathrm{~nm}$, calculated on the basis of a calibration curve, and expressed as units per liter (IU/L).

\section{Determination of oxidative stress markers in liver tissue}

Liver homogenates, obtained from homogenization of liver tissues in phosphate-buffered saline (PBS, $\mathrm{pH}$ 7.4), were used to determine the tissue levels of malondialdehyde (MDA), reduced form of glutathione (GSH), and glutathione peroxidase activity (GPx).

\section{Tissue malondialdehyde level assessment}

Hepatic concentrations of MDA were assessed as previously described (Ohkawa, Ohishi, 1979). Briefly, $200 \mu \mathrm{l}$ of liver homogenate was diluted with $800 \mu \mathrm{l}$ of PBS. $500 \mu \mathrm{l}$ of trichloroacetic acid (50\%) and $25 \mu \mathrm{l}$ of butylhydroxytoluene were mixed with the previous solution and incubated for 2 hours. After that, the mixture was centrifuged for 15 minutes, and the supernatant was mixed with $250 \mu \mathrm{l}$ of 2-thiobarbituric acid $(0.05 \mathrm{M})$ and incubated at a temperature of $95{ }^{\circ} \mathrm{C}$ to yield a pink colored TBA-reactive product. Samples were read at 532 $\mathrm{nm}$, and results are expressed in $\mathrm{nmol} / \mathrm{mL}$.

\section{Reduced glutathione assessment}

Hepatic glutathione content was measured using commercial kit (Biodiagnostic, Egypt) following the manufacturer's protocol. The method is based on the reductive cleavage of 5,5-dithiobis-2 nitrobenzoic acid by SH group to yield a yellow color. The absorbance was measured at $412 \mathrm{~nm}$ and the results were expressed as micromoles per gram tissues ( $\mu \mathrm{mol} / \mathrm{g}$ tissues).

\section{Glutathione peroxidase activity assessment}

Glutathione peroxidase (GPX) activity was assessed in the liver tissues spectrophotometrically using EnzyChrom ${ }^{\mathrm{TM}}$ Glutathione Peroxidase Assay Kit (EGPX-100). The method is based on the oxidation of $\mathrm{NADPH}$ in presence of GSH, $t$-butyl hydroperoxide, glutathione reductase (GR), and the sample with the formation oxidized glutathione (GSSG). GPX enzymatic unit was defined as the amount of the enzyme that catalyzes the conversion of one micromole of substrate per minute. The absorbance was read at $340 \mathrm{~nm}$ and GPX activity was expressed as $\mathrm{nmol} / \mathrm{min} / \mathrm{mg}$ protein.

\section{Determination of proinflammatory cytokines}

The serum tumor necrosis factor $\alpha$ (TNF- $\alpha$ ) and interleukin-1 $\beta$ (IL-1 $\beta$ ) were assayed using a commercial enzyme-linked immunosorbent assay (ELISA) kits (eBioscience, Vienna, Austria) following the manufacturer's instructions. Briefly, $50 \mu 1$ of control buffer, standard, and sample solutions were added to wells coated with the respective antibody and incubated with the diluent and biotin conjugate at room temperature. After $2 \mathrm{~h}$, wells were washed. Diluted StreptavidinHorseradish Peroxidase (HRP) was added and incubated again for another 2 hours. Wells were then washed and incubated with TMB substrate solution for 30 minutes at room temperature. To stop the enzymatic reaction, $100 \mu \mathrm{l}$ of $\mathrm{H}_{2} \mathrm{SO}_{4}$ was added, and the absorbance of the products of the reactions was read at $450 \mathrm{~nm}$. Results are expressed as picograms per milliliter $(\mathrm{pg} / \mathrm{mL})$.

\section{Hepatic-NF-E2-related factor-2 (Nrf2) assessment}

Nrf-2 quantification was determined using NFE2L2 ELISA Kit (Uscnk Biodiagnostic, USA) following the manufacturer's protocol. The test principle applied in this kit is sandwich enzyme immunoassay. Standards or samples were added to the appropriate microtiter plate wells coated with a specific biotin-conjugated antibody. Next, Avidin conjugated to HRP was added to each microplate well and incubated. After TMB substrate solution was added, wells exhibited a change in color that was measured spectrophotometrically at a wavelength of $450 \mathrm{~nm}$. The results are expressed as picograms per $\mathrm{mg}$ protein ( $\mathrm{pg} / \mathrm{mg}$ protein).

\section{Liver histological examination}

Liver tissues were fixed in a buffer solution containing $10 \%$ formalin and processed for routine paraffin block preparation. Sections of $5 \mu \mathrm{m}$ were cut 
and stained with hematoxylin and eosin stain (H\&E) for histological examination.

We applied the previously described criteria in grading the severity of the hepatic congestion or necrosis (Blazka et al., 1996). Grade 0 denotes normal histology. Grade 1 refers to minimal congestion and necrosis limited to the area surrounding the centrilobular vein. Grade 2 is characterized by extension of congestion and hemorrhage to the mid-zonal cells in most of the lobules and confluent necrosis limited to the area surrounding the centrilobular vein. Grade 3 is characterized by widespread areas of congestion and hemorrhage, and the centrilobular zone is occupied by areas of confluent necrosis with severe lobular damage.

\section{Statistical analysis of the data}

Data were analyzed using IBM SPSS software package version 20.0. (Armonk, NY: IBM Corp). The
Kolmogorov-Smirnov, Shapiro, and D'agstino tests were used to verify the normality of distribution of variables. One-way analysis of variance (one-way ANOVA) was used to compare more than two groups for normally distributed quantitative variables and followed by post hoc test (Tukey) for pairwise comparison. Results are presented as mean \pm standard error of mean (SEM). The significance of the obtained results was judged at the $5 \%$ level $(P<0.05)$.

\section{RESULTS}

\section{Effects of sitagliptin on APAP-induced alterations in serum ALT and AST levels}

Figure 1 depicts that APAP administration resulted in a significant increase in serum ALT and AST levels $(2731.4 \pm 13.5$ and $2348 \pm 11.7 \mathrm{IU} / \mathrm{L}$, resp.) as compared to the normal control group $(45.2 \pm 0.6$ and $31.4 \pm 0.9$ $\mathrm{IU} / \mathrm{L}$, resp.).

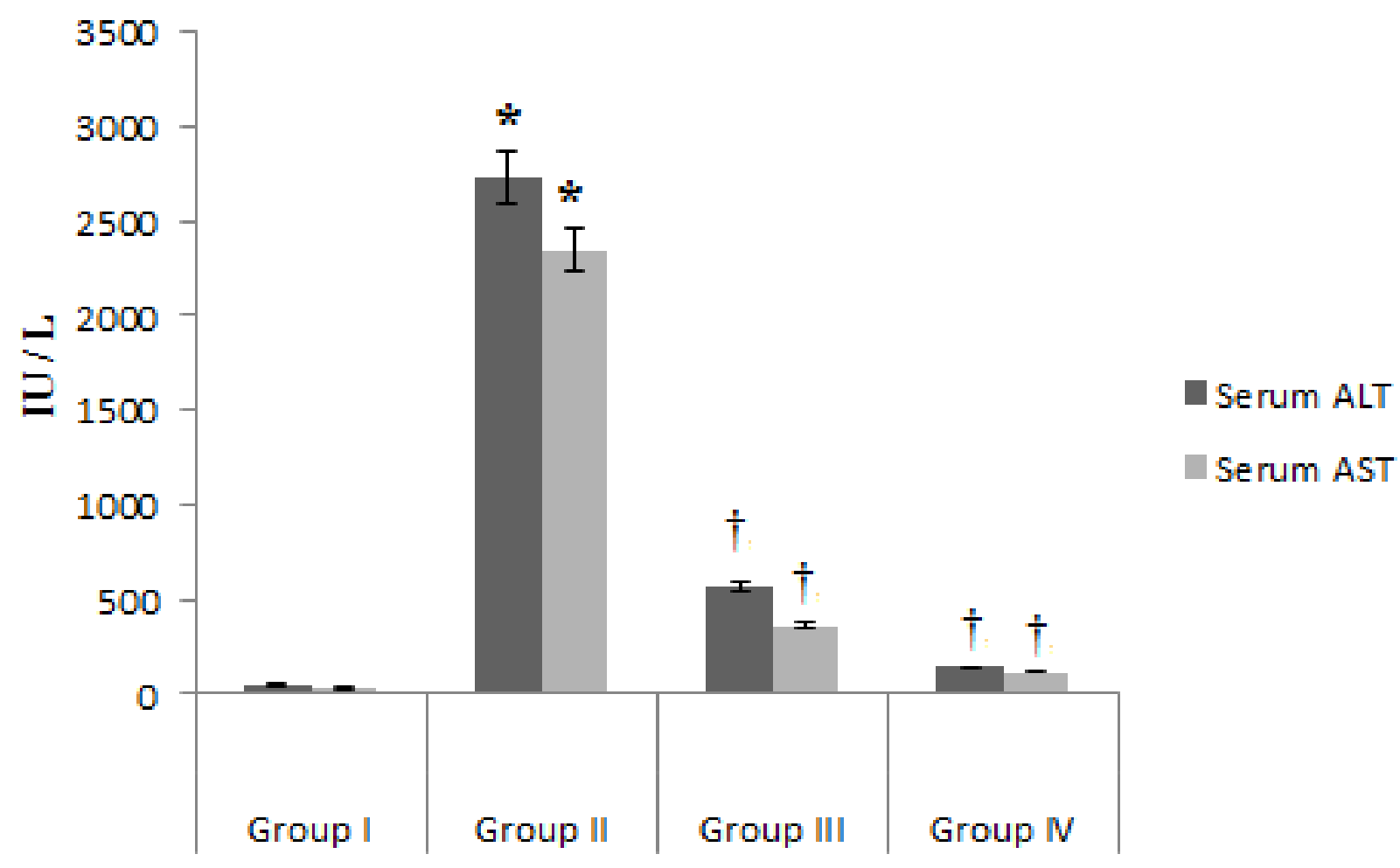

FIGURE 1 - Effects of sitagliptin on APAP-induced alterations on serum ALT (IU/L) and AST (IU/L) levels. Group I: normal control group; group II: APAP-treated group; group III: sitagliptin-treated group (10 mg/kg/day) 5 days before APAP administration; group IV: $N$-acetylcysteine-treated group $(100 \mathrm{mg} / \mathrm{kg}$ orally). Data are expressed as mean $\pm \mathrm{SEM}$ for $\mathrm{n}=10$. * $P<0.05$ compared to normal control group; $\uparrow: P<0.05$ compared to APAP control group by one-way ANOVA test followed by post hoc test. 
The increases in serum ALT and AST levels were successively attenuated by sitagliptin administration (10 mg/kg/day) for 5 days prior to APAP injection. Serum ALT was $563 \pm 4.5 \mathrm{IU} / \mathrm{L}$ and serum AST was $362 \pm 12.4 \mathrm{IU} / \mathrm{L}$ in sitagliptin treated group, $p<0.05$ as compared to APAP-treated group. $N$-Acetylcysteinetreated group showed also a significant decrease in both serum levels of AST and ALT $(137 \pm 2.4$ and $118 \pm 12.4 \mathrm{IU} / \mathrm{L}$, resp.) as compared to APAP-treated group $(p<0.05)$.

\section{Effects of sitagliptin on APAP-induced alterations in oxidative stress markers}

We evaluated hepatic oxidative status in the liver tissues by measuring malondialdehyde (MDA) content, as an end-product of lipid peroxidation, reduced glutathione, and glutathione peroxidase activity.

APAP administration resulted in an increment in MDA concentration as compared to normal control group $(146 \pm 10.3$ and $17.6 \pm 3.3 \mathrm{nmol} / \mathrm{mL}$, resp., $p<$ $0.05)$. The prophylactic sitagliptin administration, 5 days before APAP administration, and $N$-acetylcysteine treatment succeeded in suppressing MDA levels (46.5 \pm $5.3 \mathrm{nmol} / \mathrm{mL}$ and $53.7 \pm 5.2 \mathrm{nmol} / \mathrm{mL}$, resp., $p<0.05)$ as compared to the APAP-control group (Figure 2A).

GSH which is known to play an important role in the detoxification of APAP was also measured. APAP administration induced a reduction in GSH in the hepatic tissues versus the normal control group (3.7 \pm 1.2 and $9.3 \pm 1.7 \mu \mathrm{mol} / \mathrm{g}$ tissues, resp., $p<0.05$ ). On the other hand, a significant increase in GSH level was observed in both sitagliptin-treated group (8.3 $\pm 1.5 \mu \mathrm{mol} / \mathrm{g}$ tissues) and $\mathrm{N}$-acetylcysteine-treated group ( $7.1 \pm 2.1 \mu \mathrm{mol} / \mathrm{g}$ tissues) compared to the APAP control group ( $p<0.05$, Figure 2B).
Figure $2 \mathrm{C}$ depicts the effect of APAP treatment on GPx activity. APAP administration resulted in decreased level of GPx activity as compared to control group $(18.2 \pm 3$ versus $36.8 \pm 10.4 \mathrm{nmol} / \mathrm{min} /$ mg protein, $p<0.05)$. The protective administration of sitagliptin 5 days prior to APAP administration resulted in a significant increase in GPx activity (107.8 $\pm 14.5 \mathrm{nmol} / \mathrm{min} / \mathrm{mg}$ protein) as compared to normal control group and APAP-treated group ( $p<0.05$, Figure 2A). Treatment with $N$-acetylcysteine after APAP challenge produced also a significant increase in GPx activity as compared to APAP control group $(29.8 \pm 6.4, p<0.05)$; however, it did not exceed the normal control value.

\section{Effects of sitagliptin on APAP-induced alterations in inflammatory cytokines}

Figure 3 showed that the serum levels of both inflammatory cytokines TNF- $\alpha$ and IL-1 $\beta$ were significantly increased in APAP control group (82.3 \pm 5 $\mathrm{pg} / \mathrm{mL}$ and $33.7 \pm 2.2 \mathrm{pg} / \mathrm{mL}$, resp.) compared to normal control group $(23.6 \pm 4.8$ and $7.4 \pm 3.5 \mathrm{pg} / \mathrm{mL}$, resp., $p<0.05)$.

The administration of sitagliptin 5 days before APAP decreased TNF- $\alpha$ and IL- $1 \beta$ serum levels significantly $(32.9 \pm 3.7 \mathrm{pg} / \mathrm{mL}$ and $12.7 \pm 5.2 \mathrm{pg} / \mathrm{mL}$, resp.) versus APAP control group $(p<0.05)$.

$\mathrm{N}$-Acetylcysteine treatment succeeded also in counteracting the increase in TNF- $\alpha$ and IL-1 $\beta$ levels $(58.3 \pm 5.2 \mathrm{pg} / \mathrm{mL}$ and $18.4 \pm 4.1 \mathrm{pg} / \mathrm{mL}$, resp. $)$ compared to APAP control group. However, there was still a statistical significant difference between TNF- $\alpha$ serum level of the $N$-acetylcysteine-treated group and normal control group $(p<0.05)$. 

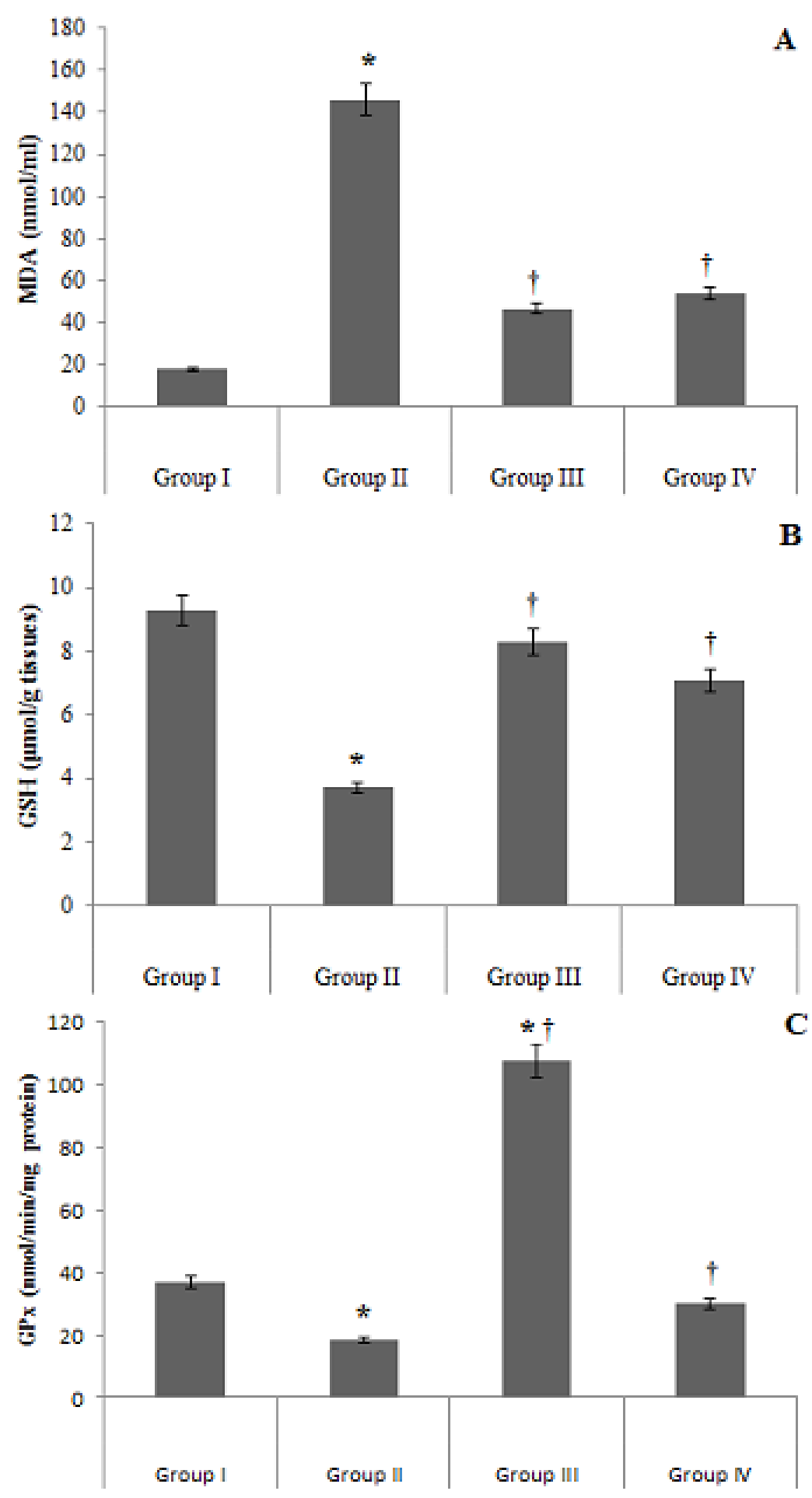

FIGURE 2 - Effects sitagliptin on APAP-induced alterations in oxidative stress markers in hepatic tissues. 2A: malondialdehyde (MDA) level; 2B: reduced glutathione (GSH) level; 2C: glutathione peroxidase (GPX) activity. Group I: normal control group; group II: APAP-treated group; group III: sitagliptin-treated group $(10 \mathrm{mg} / \mathrm{kg} / \mathrm{day}) 5$ days before APAP administration; group IV: $N$-acetylcysteine-treated group $\left(100 \mathrm{mg} / \mathrm{kg}\right.$ orally). Data are expressed as mean $\pm \mathrm{SEM}$ for $\mathrm{n}=10 .^{*}: P<0.05$ compared to normal control group; $\uparrow: P<0.05$ compared to APAP control group by one-way ANOVA test followed by post hoc test. 

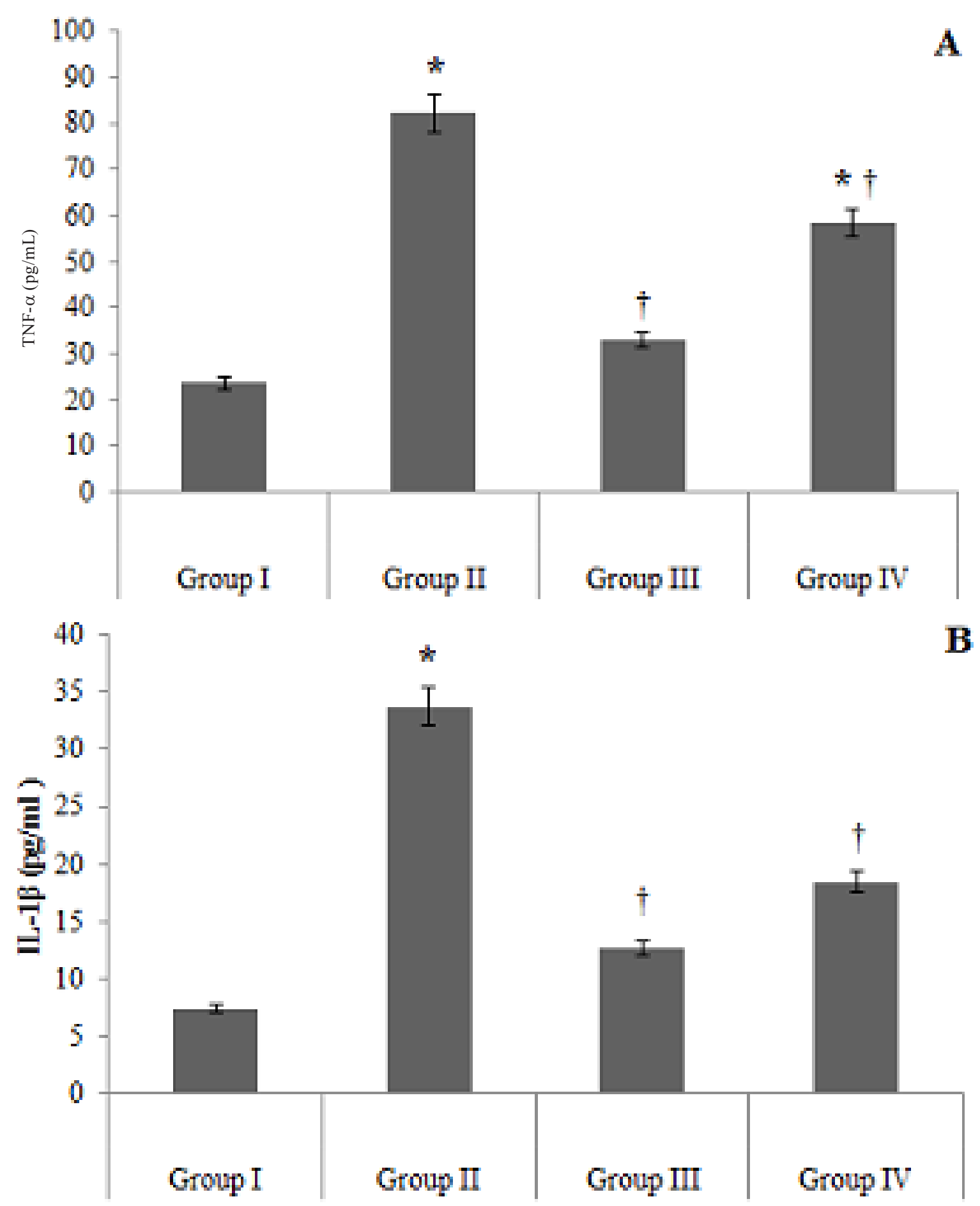

FIGURE 3 - Effects of sitagliptin on APAP-induced alterations of (A) tumor necrosis factor- $\alpha(\mathrm{pg} / \mathrm{mL})$ and (B) interleukin- $1 \beta$ $(\mathrm{pg} / \mathrm{mL})$. Group I: normal control group; group II: APAP-treated group; group III: sitagliptin-treated group (10 mg/kg/day) 5 days before APAP administration; group IV: $N$-acetylcysteine-treated group (100 $\mathrm{mg} / \mathrm{kg}$ orally). Data are expressed as mean \pm SEM for $\mathrm{n}=10$. *: $P<0.05$ compared to normal control group; $\dagger: P<0.05$ compared to APAP control group by one-way ANOVA test followed by post hoc test. 


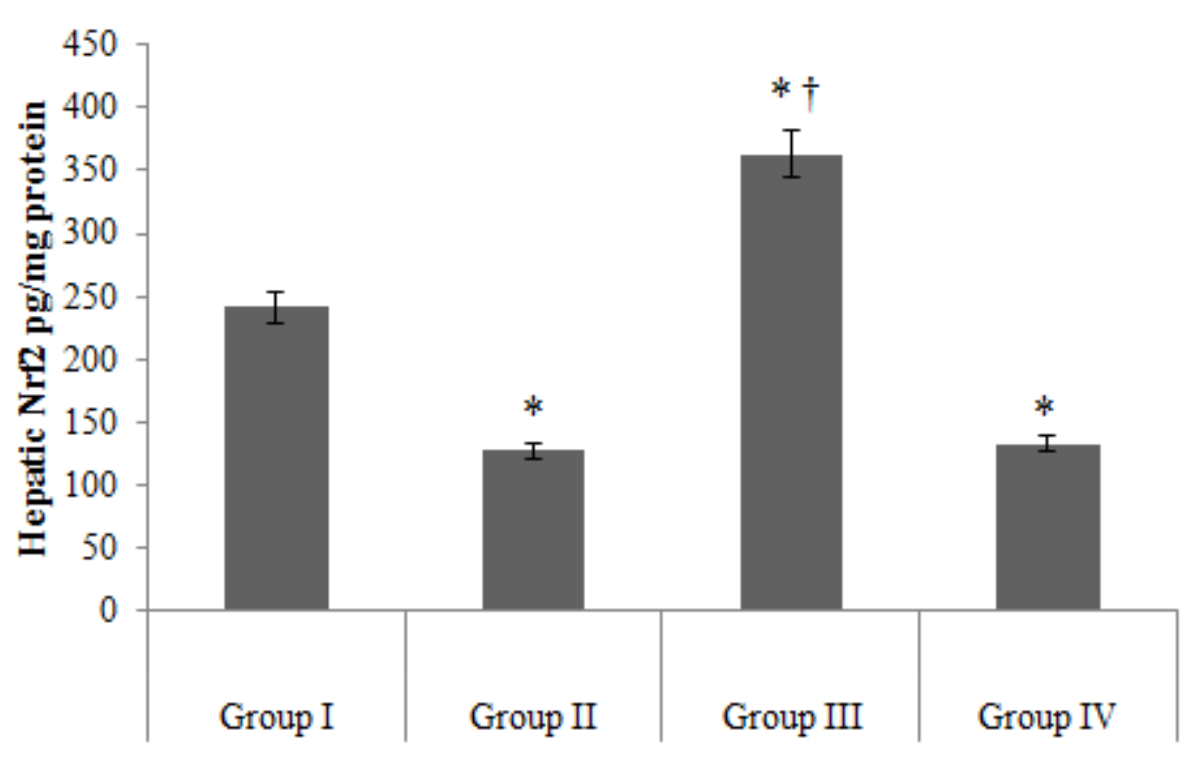

FIGURE 4 - Effects of sitagliptin on APAP-induced alterations in Hepatic Nrf2 levels (pg/mg protein). Group I: normal control group; group II: APAP-treated group; group III: sitagliptin-treated group (10 mg/kg/day) 5 days before APAP administration; group IV: $N$-acetylcysteine-treated group $(100 \mathrm{mg} / \mathrm{kg}$ orally). Data are expressed as mean $\pm \mathrm{SEM}$ for $\mathrm{n}=10$. $*: P<0.05$ compared to normal control group; $\uparrow: P<0.05$ compared to APAP control group by one-way ANOVA test followed by post hoc test.

\section{Effect of sitagliptin on APAP-induced alterations in nuclear factor erythroid 2-related factor 2 (Nrf-2) in Mice}

Nrf2 is known to function as an important transcription factor that is translocated to the nucleus and induces the transcription of genes for antioxidants or detoxification proteins. Coincident with the depression of the anti-oxidant status of the liver, APAP control group showed a statistically decreased level of hepatic Nrf2 level (127.4 $\pm 8.3 \mathrm{pg} / \mathrm{mg}$ protein) as compared to normal control group ( $241.6 \pm 5.4 \mathrm{pg} / \mathrm{mg}$ protein).

The prophylactic administration of sitagliptin, 5 days before APAP administration, resulted in a significant increase in hepatic Nrf2 level (362.9 \pm 4.8 $\mathrm{pg} / \mathrm{mg}$ protein) as compared to APAP control group as well as the normal control group ( $p<0.05$, Figure 4$)$. $\mathrm{N}$-Acetylcysteine treatment showed a nonsignificant difference in the Nrf2 level as compared to APAP control group ( $p>0.05$, Figure 4).

\section{Histological analysis}

Histological analysis of liver tissues from APAP control group confirmed APAP induced hepatotoxicity. Hepatic congestion, necrosis, and inflammatory cells infiltration were observed by $H \& E$ staining of liver tissue samples of this group (Figure 5). Treatment with sitagliptin for 5 days before APAP administration markedly reversed the APAP-induced hepatocellular damage and resulted in a reduction in the necrosis grade compared to APAP control group $(0.9 \pm 0.5$ compared to $2.4 \pm 0.7$, resp., $p<0.05$, Figure 6 ). Treatment with $\mathrm{N}$-acetylcysteine produced a reduction in the necrosis grade as compared to APAP control group $(1.2 \pm 0.7$ versus $2.4 \pm 0.7, p<0.05$ ). 

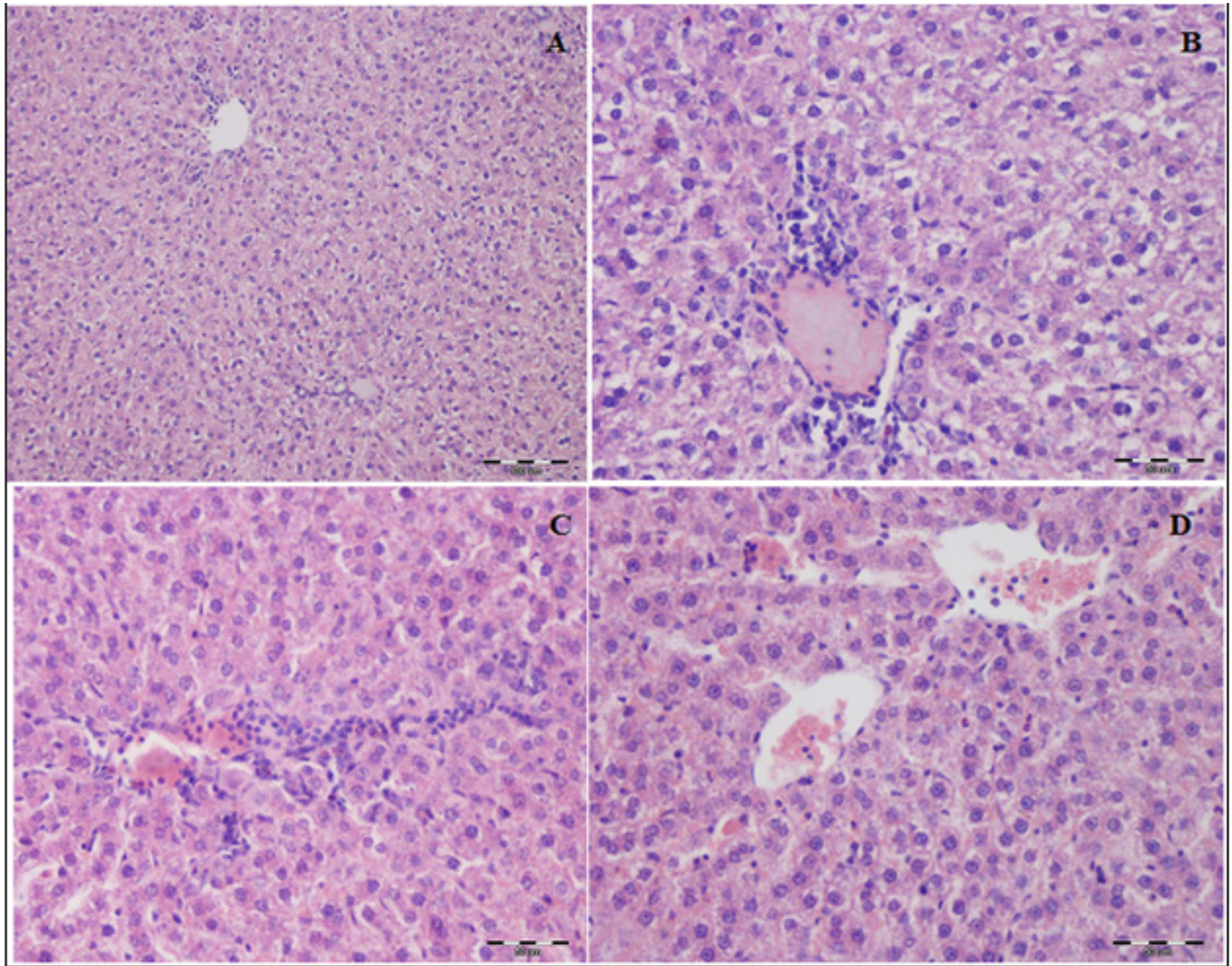

FIGURE 5 - Representative photomicrographs of liver sections from (A) normal control group showing normal liver architecture $(\mathrm{H} \& \mathrm{E} \times 200)$; (B) APAP-treated group showing hepatocyte degeneration, necrosis, congestion, and inflammatory cells infiltrations in periportal area $(\mathrm{H} \& \mathrm{E} \times 400) ;(\mathrm{C})$ sitagliptin-treated group $(10 \mathrm{mg} / \mathrm{kg} /$ day for 5 days before APAP administration $)$ showing mild congestion and hepatocyte degeneration at periportal area (H\&E $\times 400)$; (D) $N$-acetylcysteine-treated group (100 $\mathrm{mg} / \mathrm{kg}$ orally) showing amelioration of liver alterations with mild congestion and inflammatory infiltrates $(\mathrm{H} \& \mathrm{E} \times 400)$. 


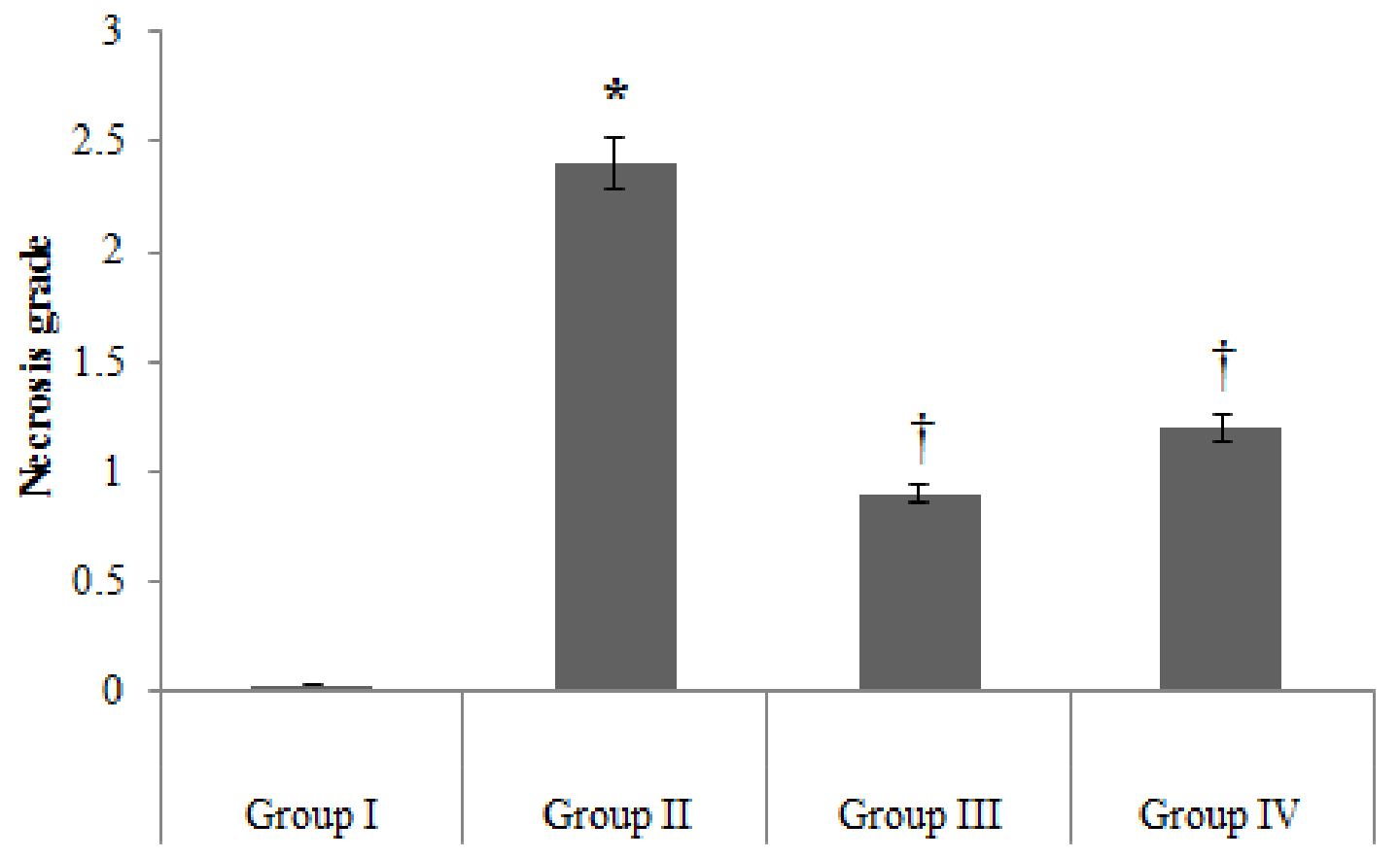

FIGURE 6 - Effects of sitagliptin on APAP-induced alterations on necrosis grade. Group I: normal control group; group II: APAP-treated group; group III: sitagliptin-treated group $(10 \mathrm{mg} / \mathrm{kg} / \mathrm{day}) 5$ days before APAP administration; group IV: $N$-acetylcysteine-treated group $(100 \mathrm{mg} / \mathrm{kg}$ orally). Data are expressed as mean $\pm \mathrm{SEM}$ for $\mathrm{n}=10$. $*: P<0.05$ compared to normal control group; $\uparrow: P<0.05$ compared to APAP control group by one-way ANOVA test followed by post hoc test.

\section{DISCUSSION}

Acetaminophen-induced hepatotoxicity has been pointed out as the most relevant example of drug induced liver injury. We aimed at the present study to evaluate the possible protective effect of sitagliptin, a DPP-4 inhibitor, against APAP-induced hepatotoxicity.

Results of the present study revealed that APAP administration resulted in marked hepatotoxicity as evidenced by a significant increase in liver transaminases associated with deteriorated histopathological grading. Leaching of both ALT and AST into the circulation reflects disturbed hepatocytes integrity and breakdown of their biomembranes which in turn is associated with hepatocyte death (Ozer et al., 2008). The interaction between the parenchymal and nonparenchymal hepatic cells produces reactive oxygen species and proinflammatory mediators responsible for the progression of tissue injury and development of hepatic necrosis (Laskin, Pilaro, Li, 1986). Concurrent with these changes, APAP administration led to an elevation in MDA level and decreased GSH level and GPx activity. The serum levels of TNF- $\alpha$ and IL- $1 \beta$ were increased, while Nrf2 level was decreased.
It is well known that APAP-induced hepatotoxicity is initiated by the metabolic activation of APAP to the highly reactive metabolite (NAPQI) that binds covalently and irreversibly to different cellular proteins causing cytotoxicity. However, it has been found that this covalent binding not to be always correlated with the severity of liver injury. The role of oxidant stress as a supplementary mechanism of cell injury was further investigated ( $\mathrm{Du}$, Ramachandran, Jaeschke, 2016). It was originally assumed that the P450-dependent metabolism of APAP is the main source of ROS formation. However, it becomes evident that selective ROS formation occurs in the mitochondria after the initial metabolism of APAP (Jaeschke, McGill, Ramachandran, 2012). NAPQI covalently binds to mitochondrial proteins. This will lead to reduction of mitochondrial respiration and ATP synthesis with subsequent generation of increased amounts of superoxide. Thus, mitochondrial dysfunction and generation of oxidative and nitrative species are critical events to amplify the cell injury mechanism (Bajt et al., 2008). Further, the interaction of free radicals with lipids enhances lipid peroxidation with an increase in serum MDA level (Alam et al., 2015). 
GSH and its related enzymes are the key antioxidant system that counteracts oxidative stress injury. It has been previously found that APAP administration results in reduction in glutathione peroxidase activity. Recently, Kanno et al. (2017) suggest that one of GPx isozymes (GPx3) is an important factor for inhibition of APAP-induced hepatotoxicity both in vivo and in vitro. Furthermore, they suggested that the higher blood GPx3 mRNA expression and plasma GPx activity in female mice may confer partial resistance to APAP toxicity in female compared to male mice.

The present study revealed that acetaminophen significantly increases TNF- $\alpha$ and IL- $1 \beta$ levels. Both of these proinflammatory cytokines are known to be involved in mediating the inflammatory response. As a support, previous studies have suggested a role of inflammatory responses in the progression of APAPinduced toxicity (Blazka et al., 1995; Martin-Murphy, Holt, Ju, 2010). The release of a variety of inflammatory mediators (macrophage migration inhibitory factor, interferon- $\gamma$, and TNF- $\alpha$ ) was found to contribute to the severity of liver injury. TNF- $\alpha$ and IL-1 are suggested to be produced by activated Kupffer cells (KCs). They stimulate recruitment of additional inflammatory mediators to the damaged areas and enhance ROS production, augmenting the hepatic damage (Blazka et al., 1995; Laskin, Pilaro, Ji, 1986).

Results of the present study showed that the APAPinduced increases in serum ALT and AST levels were drastically attenuated by sitagliptin administration 5 days before APAP injection, suggesting preservation of liver functions. The histological examination supports the ameliorative effect of pretreatment with sitagliptin on liver architecture. Moreover, sitagliptin pretreatment altered the oxidative imbalance induced by APAP challenge and decreased the serum levels of TNF- $\alpha$ and IL-1 $\beta$. N-Acetylcysteine, the gold standard for treatment of acute APAP toxicity, markedly improved the biochemical and histological findings.

The hepatoprotective effect of sitagliptin had been previously reported in several animal models of liver injury. Sitagliptin ameliorated the development of NAFLD in different animal models (Onoyama et al., 2015; Sujishi et al., 2015; Xu et al., 2017). The beneficial effects of sitagliptin were observed to be independent of blood glucose control or inhibition of fatty acids synthesis in the liver. It had been assumed that sitagliptin attenuated the progression of hepatic fibrosis due to inhibition of the inflammatory reaction and oxidative stress in animal models of NAFLD (Onoyama et al., 2015).

The pleiotropic effects of sitagliptin have also been reported in other organs. Sitagliptin modulated the antioxidant response in the diabetic kidney independent of its effect on blood glucose level (Civantos et al., 2017). It also displayed a protective effect in acute IR renal injury via suppression of ROS generation and the associated inflammatory reaction (Chang et al., 2015). Chang et al. (2013) found that sitagliptin pretreatment improved cardiac functions, increased the levels of GSH-Px and SOD, and decreased MDA level and markers of apoptosis in a rat model of myocardial I/R injury. Furthermore, sitagliptin also alleviated oxidative damage and reduced the activation of NF- $\kappa \mathrm{B}$ with a significant improvement of cardiac function in doxorubicin-induced cardiotoxicity (El-Agamy, AboHaded, Elkablawy, 2016).

Consistent with the anti-inflammatory effect of sitagliptin observed in experimental animals, Makdissi et al. (2012) demonstrated that sitagliptin exerts a potent anti-inflammatory effect in the human as reflected by suppression of the expression of a number of proinflammatory cytokines and chemokines and reduction of plasma CRP and IL-6 concentrations. Additionally, Lin and Lin reported that sitagliptin dose-dependently inhibits expression of inflammatory mediators (IL-6, IL-1 $\beta$, COX-2, and iNOS) and suppresses NF- $\kappa$ B activation in vitro (Lin, Lin, 2016).

To gain further insight into the hepatoprotective mechanisms of sitagliptin, we studied its effect on the hepatic Nrf2 level. Over the last decade, Nrf2 emerges as a major regulator in the defense against varieties of hepatotoxicants, including APAP-induced toxicity. Nrf2-deficient mice are highly susceptible to APAPinduced liver injury compared to their wild-type counterparts (Chan, Han, Kan, 2001), whereas deletion of Keap1, which degrades Nrf2, effectively inhibited acetaminophen toxicity (Okawa et al., 2006).

Nrf2 regulates a number of drug metabolizing enzymes and antioxidant genes; some of them are important for biotransformation and excretion of APAP (Chan et al., 2001; Enomoto et al., 2001). Among these enzymes are the following: $\mathrm{NAD}(\mathrm{P}) \mathrm{H}$ :quinone oxidoreductase 1 (NQO1) which is shown to reduce NAPQI back to the parent compound in vitro (Moffit et al., 2007); GCLC and GCLM which are important GSH-producing enzymes (Shin et al., 2013); the enzyme glutathione- $S$-transferase that is responsible for 
catalyzing the conjugation of toxic chemicals with GSH. Consequently, Nrf2-null mice have decreased levels and activity of hepatocellular GSH which is crucial for hepatic cells to combat oxidative stress (Ma, 2013).

In the current study, we found that sitagliptin pretreatment was associated with an increased hepatic Nrf2 level. A number of recent studies pointed to the ability of DDP4 inhibitors to target Nrf2 signaling pathway. Gemigliptin was reported to produce a direct vascular protective effect via enhanced $\mathrm{Nrf} 2$ mRNA expression and activity, leading to the induction of Nrf2 target genes (Choi et al., 2015). Sitagliptin was also reported to ameliorate methotrexate-induced hepatotoxicity via modulation of Nrf2 and NF-B signaling pathways with subsequent anti-inflammatory and antiapoptotic activity (Abo-Haded et al., 2017). Furthermore, Civantos et al. (2017) reported that sitagliptin exerts a direct renoprotective via modulation of the Nrf2 system.

In conclusion, we can speculate that sitagliptin can combat APAP-induced hepatotoxicity. The potent hepatoprotective effect of sitagliptin pretreatment could be attributed to its antioxidant and anti-inflammatory actions and modulation of Nrf2 in hepatic tissues. Future studies addressing the role of sitagliptin in APAP-toxicity in special circumstances, such as obese, diabetic, and fatty liver animal models, need to be investigated.

\section{REFERENCES}

Abo-Haded H, Elkablawy M, Al-Johani Z, Al-AhmadiO, El-Agamy D. Hepatoprotective effect of sitagliptin against methotrexate induced liver toxicity. PLoS One. 2017;12(3):e0174295.

Alam M, Hasan Chowdhury M, Jain P, Sagor M, Reza H. DPP-4 inhibitor sitagliptin prevents inflammation and oxidative stress of heart and kidney in two kidney and one clip (2K1C) rats. Diabetol Metab Syndr. 2015;7:107.

Bajt M, Farhood A, Lemasters J, Jaeschke H. Mitochondrial bax translocation accelerates DNA fragmentation and cell necrosis in a murine model of acetaminophen hepatotoxicity. J Pharmacol Exp Ther. 2008;324(1):8-14.

Blazka M, Elwell M, Holladay S, Wilson R, Luster M. Histopathology of acetaminophen-induced liver changes: role of interleukin $1 \alpha$ and tumor necrosis factor $\alpha$. Toxicol Pathol. 1996;24(2):181-9.
Blazka M, Wilmer J, Holladay S, Wilson R, Luster M. Role of proinflammatory cytokines in acetaminophen hepatotoxicity. Toxicol Appl Pharmacol. 1995;133(1):43-52.

Chan K, Han X, Kan Y. An important function of Nrf2 in combating oxidative stress: detoxification of acetaminophen. Proc Natl Acad Sci U S A. 2001;98(8):4611-6.

Chang G, Zhang P, Ye L, Lu K, Wang Y, Duan, et al. Protective effects of sitagliptin on myocardial injury and cardiac function in an ischemia/reperfusion rat model. Eur J Pharmacol. 2013;718(1-3):105-13.

Chang M, Chen C, Chen Y, Wu Y, Zhen Y, Leu S, et al. Sitagliptin protects rat kidneys from acute ischemiareperfusion injury via upregulation of GLP-1 and GLP-1 receptors. Acta Pharmacol Sin. 2015;36(1):119-30.

Choi S, Park S, Oh C, Leem J, Park KG, Lee IK. Dipeptidyl peptidase-4 inhibition by gemigliptin prevents abnormal vascular remodeling via NF-E2-related factor 2 activation. Vascul Pharmacol. 2015;73:11-9.

Civantos E, Bosch E, Ramirez E, Zhenyukh O, Egido J, Lorenzo O, et al. Sitagliptin ameliorates oxidative stress in experimental diabetic nephropathy by diminishing the miR200a/Keap-1/Nrf2 antioxidant pathway. Diabetes Metab Syndr Obes. 2017; 10:207-22.

Dambach D, Durham S, Laskin J, Laskin D. Distinct roles of NF-kappaB p50 in the regulation of acetaminophen-induced inflammatory mediator production and hepatotoxicity. Toxicol Appl Pharmacol. 2006;211(2):157-65.

Du K, Ramachandran A, Jaeschke H. Oxidative stress during acetaminophen hepatotoxicity: Sources, pathophysiological role and therapeutic potential. Redox Biol. 2016;10:148-56.

El-Agamy D, Abo-Haded H, Elkablawy M. Cardioprotective effects of sitagliptin against doxorubicin-induced cardiotoxicity in rats. Exp Biol Med. 2016;241(14):1577-87.

Enomoto A, Itoh K, Nagayoshi E, Haruta J, Kimura T, O'Connor T, et al. High sensitivity of Nrf2 knockout mice to acetaminophen hepatotoxicity associated with decreased expression of ARE-regulated drug metabolizing enzymes and antioxidant genes. Toxicol Sci. 2001;59(1):169-77.

Jaeschke H, Knight T, Bajt M. The role of oxidant stress and reactive nitrogen species in acetaminophen hepatotoxicity. Toxicol Lett. 2003;144(3):279-88.

Jaeschke H, McGill M, Ramachandran A. Oxidant stress, mitochondria, and cell death mechanisms in druginduced liver injury: lessons learned from acetaminophen hepatotoxicity. Drug Metab Rev. 2012;44(1):88-106. 
Jung Y, Choi Y, Jung G, Seo H, Kim H, Jang B, et al. Sitagliptin attenuates methionine/choline-deficient diet-induced steatohepatitis. Diabetes Res Clin Pract. 2014;105(1):47-57.

Kanno S, Tomizawa A, Yomogida S, Hara A. Glutathione peroxidase 3 is a protective factor against acetaminopheninduced hepatotoxicity in vivo and in vitro. Int $\mathrm{J}$ Mol Med. 2017;40(3):748-54.

Lancaster E, Hiatt J, Zarrinpar A. Acetaminophen hepatotoxicity: an updated review. Arch Toxicol. 2015;89(2):193-9.

Laskin D, Pilaro A, Ji S. Potential role of activated macrophages in acetaminophen hepatotoxicity. II. Mechanism of macrophage accumulation and activation. Toxicol Appl Pharmacol. 1986;86(2):216-26.

Lee J, Li J, Johnson D, Stein TD, Kraft AD, Calkins M, et al. Nrf2, a multi-organ protector? FASEB J. 2005;19(9):1061-6.

Lin C, Lin C. Sitagliptin attenuates inflammatory responses in lipopolysaccharide-stimulated cardiomyocytes via nuclear factor- B pathway inhibition. Exp Ther Med. 2016;11(6):2609-15.

Ma Q. Role of Nrf2 in oxidative stress and toxicity. Annu Rev Pharmacol Toxicol. 2013;53:401-26.

Makdissi A, Ghanim H, Vora M, Green K, Abuaysheh S, Chaudhuri A, et al. Sitagliptin Exerts an Antinflammatory Action. J Clin Endocrinol Metab. 2012;97(9):3333-41.

Martin-Murphy B, Holt M, Ju C. The role of damage associated molecular pattern molecules in acetaminopheninduced liver injury in mice. Toxicol Lett. 2010;192(3): $387-94$.

McGill M, Jaeschke H. Metabolism and disposition of acetaminophen: recent advances in relation to hepatotoxicity and diagnosis. Pharm Res. 2013;30(9):2174-87.

Michaut A, Moreau C, Robin MA, Fromenty B. Acetaminophen-induced liver injury in obesity and nonalcoholic fatty liver disease. Liver Int. 2014;34(7):171-9.

Moffit J, Aleksunes L, Kardas S, Klaassen C, Manautou J. Role of NAD(P)H:quinone oxidoreductase 1 in clofibratemediated hepatoprotection from acetaminophen. Toxicology. 2007;230(2-3):197-206.

Mulvihill E, Drucker D. Pharmacology, physiology, and mechanisms of action of dipeptidyl peptidase-4 inhibitors. Endocr Rev. 2014; 35(6):992-1019.
Ohkawa H, Ohishi N. Assay for lipid peroxides in animal tissues by thiobarbituric acid reaction. Anal Biochem. 1979;95(2):351-8.

Okawa H, Motohashi H, Kobayashi A, Aburatani H, Kensler TW, Yamamoto M. Hepatocyte-specific deletion of the keapl gene activates Nrf2 and confers potent resistance against acute drug toxicity. Biochem Biophys Res Commun. 2006;339(1):79-88.

Onoyama T, Koda M, Okamoto T, Kishina M, Matono $\mathrm{T}$, Sugihara T, et al. Therapeutic effects of the dipeptidyl peptidase-IV inhibitor, sitagliptin, on non-alcoholic steatohepatitis in FLS-ob/ob male mice. Mol Med Rep. 2015;12(5):6895-902.

Ozer J, Ratner M, Shaw M, Bailey W, Schomaker S. The current state of serum biomarkers of hepatotoxicity. Toxicology. 2008;245(3):194-205.

Portillo-Sanchez P, Cusi K. Treatment of Nonalcoholic Fatty Liver Disease (NAFLD) in patients with Type 2 Diabetes Mellitus. Clin Diabetes Endocrinol. 2016;2:9.

Reitman S, Frankel S. A colorimetric method for the determination of serum glutamic oxalacetic and glutamic pyruvic transaminases. Am J Clin Pathol. 1957;28(1):56-63.

Shimizu D, Ishitsuka Y, Miyata K, Tomishima Y, Kondo $\mathrm{Y}$, Irikura $\mathrm{M}$, et al. Protection afforded by pre- or posttreatment with 4-phenylbutyrate against liver injury induced by acetaminophen overdose in mice. Pharmacol Res. 2014;87:26-41.

Shin S, Yang J, Ki S. Role of the Nrf2-ARE pathway in liver diseases. Oxid Med Cell Longev. 2013;2013:763257.

Sujishi T, Fukunishi S, Ii M, Nakamura K, Yokohama K, Ohama H, et al. Sitagliptin can inhibit the development of hepatic steatosis in high-fructose diet-fed ob/ob mice. J Clin Biochem Nutr. 2015;57(3):244-53.

Watelet J, Laurent V, Bressenot A, Bronowicki J, Larrey D, Peyrin-Biroulet L. Toxicity of chronic paracetamol ingestion. Aliment Pharmacol Ther. 2007;26(11-12):1543-4.

Xu B, Shen T, Chen L, Xia J, Zhang C, Wang H, et al. The effect of sitagliptin on lipid metabolism of fatty liver mice and related mechanisms. Med Sci Monit. 2017;23:1363-70.

Received for publication on $20^{\text {th }}$ June 2018 Accepted for publication on $07^{\text {th }}$ January 2019 